Crack-free direct-writing on glass using a low-power UV laser in the manufacture of a microfluidic chip

This article has been downloaded from IOPscience. Please scroll down to see the full text article.

2005 J. Micromech. Microeng. 151147

(http://iopscience.iop.org/0960-1317/15/6/005)

The Table of Contents and more related content is available

Download details:

IP Address: 140.112.113.225

The article was downloaded on 31/12/2008 at 03:28

Please note that terms and conditions apply. 


\title{
Crack-free direct-writing on glass using a low-power UV laser in the manufacture of a microfluidic chip
}

\author{
Ji-Yen Cheng ${ }^{1}$, Meng-Hua Yen ${ }^{1,2}$, Cheng-Wey Wei ${ }^{1,2}$, \\ Yung-Chuan Chuang ${ }^{1}$ and Tai-Horng Young ${ }^{2}$ \\ ${ }^{1}$ Research Center for Applied Sciences, Academia Sinica, Taiwan \\ ${ }^{2}$ Institute of Biomedical Engineering, National Taiwan University, Taiwan \\ E-mail: jycheng@gate.sinica.edu.tw
}

Received 16 December 2004, in final form 31 March 2005

Published 22 April 2005

Online at stacks.iop.org/JMM/15/1147

\begin{abstract}
Glass is an excellent material for use as a microfluidic chip substrate because it has great chemical and thermal stability. This work describes a flexible platform for the rapid prototyping of microfluidic chips fabricated from glass. A debris-free laser direct-writing technology that requires no photomask generation is developed. A $266 \mathrm{~nm}$ laser with a high repetition rate is employed in laser-induced backside wet etching (LIBWE) for glass machining. A microfluidic pattern is designed using computer drawing software and then automatically translated into computer numerical control motion so that the microtrench is directly fabricated on the glass chip. The overall machining speed can be increased by increasing the repetition rate to $\sim 6 \mathrm{kHz}$. Without a clean room facility or the highly corrosive acid, HF, the overall development time is within hours. Trenches with complex structures that are hard to fabricate by photolithography were easily produced by laser direct-writing. An integrated microreactor/concentrator is demonstrated. The crack-free and debris-free surface was characterized by SEM and a surface profiler. Various effective etching chemicals for the LIBWE process were investigated to understand the etching mechanism. The minimal laser power used for glass etching was approximately $20 \mathrm{~mW}$ for a $6 \mu \mathrm{m}$ wide microtrench. Several new compounds have been demonstrated to be effective in ablation. The etch threshold is minimum and does not decrease further as the unit length absorbance increases above 8000 in acetone solution.
\end{abstract}

\section{Introduction}

Numerous applications have been developed using miniaturized on-chip reactors. These include, but are not limited to, the preparation of nanoparticles [1], the preparation of highly active intermediates [2-4], the polymerase chain reaction (PCR) $[5,6]$ and catalytic reactions [7]. The substrates include silicon, quartz, glass and plastics.

Glass has excellent chemical and thermal stability. For instance, microreactors used for the Berthelot reaction and the Wittig reaction [8], the diazonium reaction [3,9] and the Suzuki reaction [7], which use chemicals that are corrosive to silicon or/and plastics, have been demonstrated on glass-based chips [10]. Moreover, glass is less able to adsorb protein than other materials, such as silicon $(\mathrm{Si})$ and silicon nitride $\left(\mathrm{Si}_{3} \mathrm{~N}_{4}\right)$, that are used extensively in chip fabrication. $\mathrm{SiO}_{2}$-coated chips have been reported to yield greater DNA amplification than the $\mathrm{Si}$ - and $\mathrm{Si}_{3} \mathrm{~N}_{4}$-coated chips [11].

Almost all of these studies on chemical and biological applications implement unique channel designs to meet the needs of each experiment. Iterated modifications are often made during the development of microfluidic chips to test a new idea experimentally. However, repeating the photomask design and photolithography process can be time-consuming. 
Having a rapid prototyping method for flexibly and quickly fabricating chips would thus be very helpful. Laser directwriting for microfluidic chips provides this advantage. This method does not require the generation of a photomask. Hence, the microfluidic pattern can be very quickly redesigned and re-fabricated. For example, the method reported in this work can fabricate a $100 \mu \mathrm{m}$ wide, $10 \mu \mathrm{m}$ deep and $100 \mathrm{~mm}$ long trench in $12 \mathrm{~min}$. The overall development time for a new prototype can be reduced from weeks to hours. Furthermore, this approach raises the fabrication capability beyond that which can be achieved or, at least, easily achieved by the extensively used photolithography process.

Laser direct-writing can be used to fabricate several structures that are hard to fabricate by photolithography. For instance, fabricating multiple trenches with different aspect ratios requires photolithography with many steps. The entire procedure requires the alignment between the substrate and the photomask. Such a structure can be easily fabricated on a single substrate by direct-writing laser ablation [12] because the trench width and depth can be varied by controlling the laser machining parameters, including the beam size and the scanning speed. Deep trenches with an aspect ratio larger than $1 / 2$, which is the practical limit for isotropic wet etching, can also be obtained easily.

The laser direct-writing method offers several other advantages. The method can be used to prepare a smooth slope easily by varying the beam sweeping speed during ablation. The production of this structure depends on the use of an expensive gray scale photomask in photolithography. Moreover, the laser beam profile can be shaped to generate a trench with corresponding cross-section.

Laser direct-writing on various types of substrate has attracted ongoing attention. Its use in the micromachining of Si [13] and various plastics [12, 14, 15] has been reported. Swift fabrication has substantially reduced the development time of the microfluidic systems. However, to the authors' knowledge, direct-writing on glass was rarely reported [16].

Numerous reports have described photomask-based or single-point laser micromachining on glass using nanosecond lasers with a high energy density $\left(3.4-5.4 \mathrm{~J} \mathrm{~cm}^{-2}\right)$ or ultrafast femtosecond lasers [17-27]. Nevertheless, only in recent years have fused silica [28-36] and glass [35] of high quality been obtained using nanosecond lasers, which are more economic than femtosecond lasers. These novel etching approaches result in crack-free and debris-free surfaces. The vacuum UV (VUV, $157 \mathrm{~nm}$ ) laser is used in crack-free glass machining [32], and two novel methods are used in high-quality etching. Both methods are suitable for etching a substrate that is transparent to the incident laser light.

Zhang et al [28] utilized an excimer laser to energize a metal target plate in proximity to a fused quartz substrate. This method requires a piece of metal target that encompasses the machining area. Laser-induced backside wet etching (LIBWE), developed by Wang et al [30], utilizes an excimer laser to excite a sensitizer solution that is in contact with the substrate. This method uses very low pulse energy for etching. The etch threshold $\left(\sim 250 \mathrm{~mJ} \mathrm{~cm}^{-2}\right)$ is approximately $1 / 40$ of that used for directly etching fused silica in air. This approach has only been tested with an excimer laser. However, the low pulse energy can easily be obtained by a diode-pumped solid state (DPSS) Nd:YAG laser.
Ding et al [33] employed a high-pressure 'liquid hammer' model to elucidate the LIBWE process. In this model, the incident laser pulse energy ( $\sim 30 \mathrm{~ns}$ pulse width) is absorbed by the solute and transferred to the solvent by rapid thermal relaxation. This photothermal process eventually generates a rapidly expanding liquid jet. The liquid jet then exerts high pressure on the substrate, resulting in the etching. Vass et al [37] very recently reached a similar conclusion, using a new sensitizer naphthalene-methyl-methacrylate. Bohme et al found the effect of different solvents on the etching threshold of fused silica in the LIBWE process [34]. Their findings support the claim that the solvent and the solute are both important to the etching mechanism.

This study uses a UV laser with a high repetition rate to test the micromachining capability of the LIBWE process. The backside etching approach has only been implemented [29, 30, $33-35,38$ ] with an excimer laser that has a low repetition rate (tens of $\mathrm{Hz}$ ). Whether a laser with a high repetition rate (tens of $\mathrm{kHz}$ ), such as the frequency-quadrupled DPSS laser, is appropriate for swift backside glass micromachining is worthy of investigation.

An $X-Y-Z$ scanning stage has been used in an earlier work on backside glass etching [35], although it has been used with a photomask, and no direct-writing has been reported. The aim of this work is to take advantage of the high repetition and easeof-maintenance of the DPSS laser to explore its application for the rapid prototyping of glass microfluidic chip. In this microfabrication system, the UV laser is incorporated with a computer numerical control (CNC) stage. The microfluidic pattern is designed using common computer software and then translated into computer-controlled motion, while the laser beam is used to etch the substrate. This combination could write the microfluidic trenches directly onto the glass chip. This work demonstrates trenches with complex features. It also demonstrates an example pattern that integrates a microreactor and a microconcentrator for on-chip chemical reactions. The high quality of machining was examined using SEM and a surface profiler. To our knowledge, this is the first work to report direct-writing on glass using a UV laser for microfluidic chip applications.

This work also compares various organic compounds to determine their effectiveness in the LIBWE process and to elucidate the etching mechanism. An attempt was made to correlate the etching threshold with the optical characteristics of these compounds.

\section{Experimental details}

\subsection{Chemical and borofloat glass substrate}

Chemicals were obtained from Acros and used without further purification. Organic solvents were acquired from Tedia or J T Baker. All the solvents were HPLC grade. The glass substrate was Borofloat 33 from Schott with a thickness of $0.7 \mathrm{~mm}$.

\subsection{Glass micromachining by backside UV laser etching}

Figure 1 depicts the laser etching setup. The frequencyquadrupled Q-switched DPSS Nd:YAG laser was obtained from Photonics Industries (266 nm, Model: DS-10E 266H). 


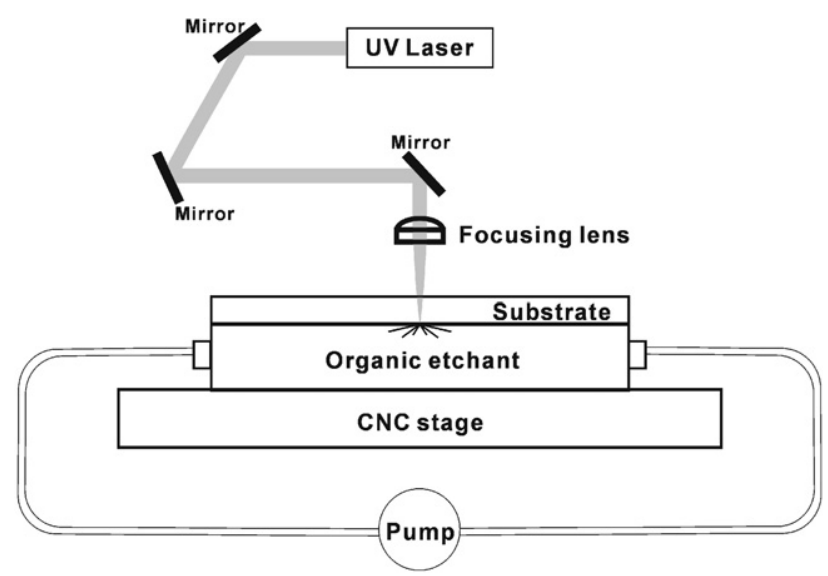

Figure 1. Setup of a DPSS laser direct-writing system for glass micromachining.

The pulse repetition rate was adjustable from $1 \mathrm{~Hz}$ to $50 \mathrm{kHz}$. The pulse width was $10 \mathrm{~ns}$ at a repetition rate of below $10 \mathrm{kHz}$. From $10 \mathrm{kHz}$ to $20 \mathrm{kHz}$, the pulse width increases gradually to $15 \mathrm{~ns}$. The maximal average power was $1.5 \mathrm{~W}$ at $10 \mathrm{kHz}$. The maximal pulse energy was $250 \mu \mathrm{J}$ when the laser was operated at $6 \mathrm{kHz}$. However, usually the energy of $78 \mu \mathrm{J}$ at $4.5 \mathrm{kHz}$ was used to generate a $30 \mu \mathrm{m}$ wide trench while $4 \mu \mathrm{J}$ was used to form a $6 \mu \mathrm{m}$ wide trench. It was measured that the borofloat absorbed $50 \%$ of the incident laser wavelength (data not shown). With such low pulse energy, the energy absorbed by the substrate does not induce etching. The diameter of the output laser beam is $0.7 \mathrm{~mm}$. The laser beam is directed perpendicular to a piece of borofloat glass that is mounted on a circulation chamber. The glass is in contact with the organic etchant, which is circulated using a pump. The glass and the circulation chamber are mounted together on a twodimensional CNC motion stage.

A glass microfluidic chip was fabricated according to the procedure described below. The microfluidic pattern was designed using CorelDraw (Corel) or AutoCAD (Autodesk). The pattern was then sent to the CNC stage to perform direct machining on the glass substrate. The laser beam is fixed while the CNC stages move as dictated by the pattern. The beam is thus swept over the glass substrate for machining. The $\mathrm{CNC}$ stage has a repeatability of $0.1 \mu \mathrm{m}$ and the straightness of the motion is $1 \mu \mathrm{m}$ per $100 \mathrm{~mm}$. After machining, the microtrench was sealed by thermally bonding the trenched substrate with a piece of blank borofloat glass. The thermal course was as follows. The sample was heated from room temperature to $600{ }^{\circ} \mathrm{C}$ at $10^{\circ} \mathrm{C} \mathrm{min}^{-1}$, and then slowly heated to $640{ }^{\circ} \mathrm{C}$ at $5^{\circ} \mathrm{C} \mathrm{min}^{-1}$, at which it was kept for $3.5 \mathrm{~h}$. The oven power was then turned off and the sample was allowed to cool to room temperature in the oven. The cooling time is about $10 \mathrm{~h}$.

A single lens is used to focus the laser beam onto the substrate. The resultant beam size is calculated according to the formula [39]

$$
d_{0}=2 F \lambda / d_{\mathrm{i}},
$$

where $d_{0}$ represents the focused beam size, $F$ is the focal length, $\lambda$ is the laser light wavelength and $d_{\mathrm{i}}$ is the initial laser beam size. The minimal focused beam size is $6 \mu \mathrm{m}$. The actual ablated feature size can be controlled by varying the distance from the focusing lens to the substrate. The feature size is then used to calculate the etching rate (nm per pulse) according to the equation

$$
R_{\text {etch }}=\frac{D}{\left(d_{0} / V\right) \times f},
$$

where $D$ is the etched depth, $V$ is the sweeping speed of the laser beam, $d_{0}$ is the beam size and $f$ is the laser repetition rate. A surface profiler (Model AS-IQ, KLA-Tencor) is employed to measure the cross-sections of the microtrenches and the longitudinal profile along the bottom thereof. The stylus has a tip angle of $60^{\circ}$. The SEM images were obtained using a Leo 1530 field emission scanning electron microscope (SEM, Leo, UK).

\section{Result and discussion}

\subsection{Asymmetric trench structure}

This work presented some examples to demonstrate the unique micromachining capability of the direct-writing approach. Figure 2 presents the first example. The three-dimensional drawing in figure $2(a)$ presents the cut view of a stepwise bottom in a trench. Fabricating this type of structure by photolithography depends on an alignment and etching process with several steps. Figure $2(b)$ presents the longitudinal stepwise profile of a glass trench fabricated by laser directwriting. The machining process is simple. A laser beam is programmed to sweep over the desired position at a preset number of passes to generate the stepwise structure. Increasing the number of passes deepens the trench. In figure $2(b)$, the etching depths for two, four and six passes are $5 \mu \mathrm{m}, 10 \mu \mathrm{m}$ and $15 \mu \mathrm{m}$, respectively. Such a structure can be used in many kinds of microdevices. For instance, the structure can be used to retain microbeads or resin to form a micro-chromatographic column [40, 41]. Such a microcolumn can be used for rapid purification of minute sample; for example, the purification column can be integrated with capillary electrophoresis for DNA sequencing [42]. Additionally, the structure can be used in microchannel cell culture system to retain cells with a diameter of $\sim 10 \mu \mathrm{m} \mathrm{[43].}$

Figure 2(c) presents another example of a complex structure. The three-dimensional illustration presents a transversely asymmetric trench. Two adjacent trenches are fabricated by sweeping two lines that are separated by a distance that is less than the width of one trench. Figure $2(d)$ reveals that the width of a single trench is $30 \mu \mathrm{m}$ and that the two adjacent trenches are separated by $24 \mu \mathrm{m}$. The measured profile presents a pair of adjacent trenches with different aspect ratios. The depths are $6 \mu \mathrm{m}$ and $12 \mu \mathrm{m}$, respectively. A shallow depth is used so that the profiler stylus can reach the bottom of the trench and detect the correct profile.

Such a structure is hard to fabricate by wet etching because the wet etching process can only produce structures with the same aspect ratio in a single process. Hence, the steps of the lithographic process must be repeated for such structures. The multiple steps of photolithography are also required to generate this asymmetric profile by dry etching, such as reactive ion etching (RIE). 


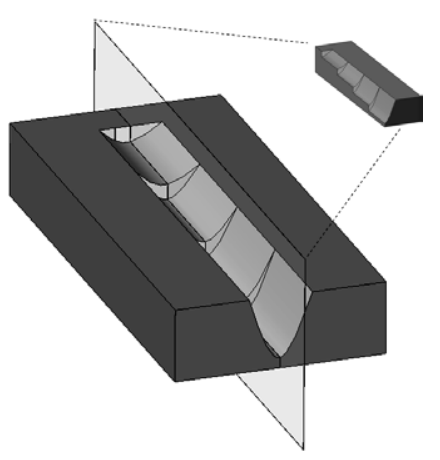

(a)

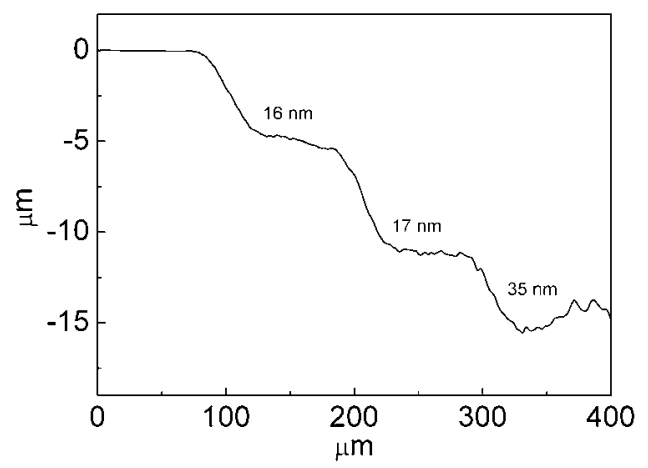

(b)

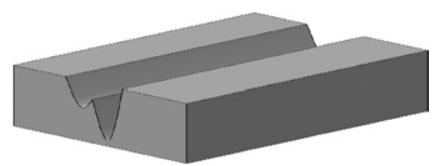

(c)

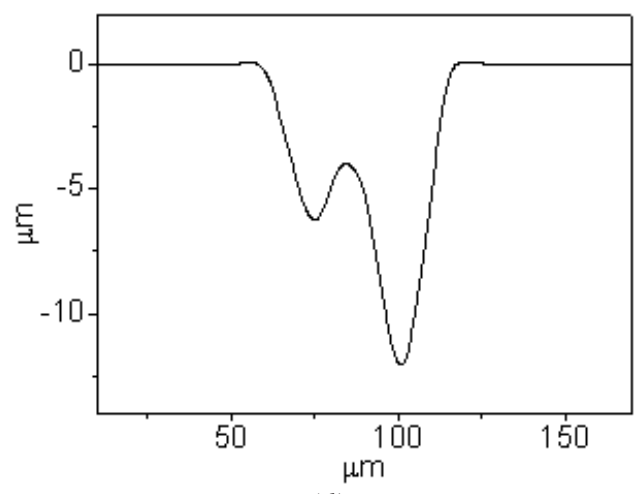

(d)

Figure 2. Examples of two types of asymmetric trench. (a) Cut-view of the three-dimensional illustration of a stepwise microstructure. (b) Longitudinally asymmetric profile along the bottom of the stepwise trench. The corresponding roughness (RMS value) for each step is as indicated. (c) Cross-section of a transversely asymmetric trench. $(d)$ Cross-section of an asymmetric trench measured by a surface profiler. The trenches in these images are etched with a laser pulse of energy $78 \mu \mathrm{J}$. The repetition rate is $4.5 \mathrm{kHz}$ and the laser sweep speed is $0.5 \mathrm{~mm} \mathrm{~s}^{-1}$ in $(b)$ and $0.2 \mathrm{~mm} \mathrm{~s}^{-1}$ in $(d)$. The etchant is $0.4 \mathrm{M}$ pyrene in acetone. The $Y$-axes in $(b)$ and $(d)$ are enlarged to show the smooth trench edge.

Figure 2(d) also presents the negligible rim distortion at the trench edge. The rim height is smaller than $0.1 \mu \mathrm{m}$, indicating that the thermal effect, if it exists, is very small during ablation. The small rim distortion is unlikely to interfere with thermal bonding with another blank substrate, as discussed below.

\subsection{Maskless and debris-free micromachining by direct-writing on glass using a laser}

The maskless direct-writing method produces debris-free and crack-free surfaces on borofloat glass. The surface profile shown in figure $2(b)$ presents the effect on the surface roughness of repeated sweeping of the laser. This profile consists of four flat stages. The leftmost one is the original borofloat surface. The surface roughness is $1.5 \mathrm{~nm}$ (RMS). Starting from this stage are three consecutive stages that are etched by two, four and six laser beam passes. Their surface roughness is $16 \mathrm{~nm}, 17 \mathrm{~nm}$ and $35 \mathrm{~nm}$ (RMS), respectively. That laser ablation roughens the surface is clear. However, the surface is smoother than the $\mathrm{Ar}^{+}$ion-etched surface that has a roughness of 550-760 $\mathrm{nm}(\mathrm{Ra})$ [44]. The result is comparable to other backside etching results [38], which reveal a roughness of approximately $30 \mathrm{~nm}$ (RMS). The roughness in figure $2(d)$ is less than that in figure $2(b)$ because the cross-section is measured using a stylus to scan perpendicular to the trench, or parallel to the ripples. The ripples are formed by the unstable velocity of the CNC stage and not by the LIBWE process (see below).

The crack-free surface was also observed under an electron microscope. Figure 3(a) presents an SEM image of the two adjacent trenches with different aspect ratios. The asymmetric structure corresponds to that shown in figures 2(c) and $(d)$. In figure $3(a)$, the right side of the trench is the deeper side. The bottom of the trench has some ripples. The ripples exhibit periodical fluctuation. The tuning of the motion parameter of the CNC stage is therefore considered. It is found that the ripples are caused by the fluctuation in the speed of the $\mathrm{CNC}$ stage. Given a constant laser pulse energy, the unstable speed makes the etching depth uneven. Optimizing the motion parameter of the CNC stage, e.g. damping level at low speed, motor inductance setting, etc, yields a very smooth surface, as shown in figures $3(b)$ and $(c)$. The magnification in figure $3(b)$ is greater than that in figure $3(a)$.

Deeper trenches can be fabricated by increasing the laser pulse energy. Figure 3(c) shows that the channel width is $10 \mu \mathrm{m}$ and the aspect ratio exceeds 0.5 . The aspect ratio can be increased by repeated ablation. Figure $3(d)$ presents a trench with an aspect ratio of larger than 2. Notably, trenches with higher aspect ratios tend to contain some debris near the sidewalls, formed probably by the re-deposition of glass during ablation. However, no crack is observed in such deep trenches. Similar to that observed in figure $2(b)$, deeper trench shows rougher surface. The direct-writing method simultaneously produces trenches with various aspect ratios on a single substrate. A photoresist layer need not be recast to define areas with different depths. Trenches with different aspect ratios can be formed by changing the laser beam pulse, the beam scanning speed or the number of beam scans. Figure 3(e) presents a chip with trenches with four aspect ratios.

Recently, a femtosecond laser direct-writing method for fabricating microfluidic chip has been reported [16]. Compared to this method, the $\mathrm{mW}$ Q-switched nanosecond laser used in our approach is more available. 
(a)
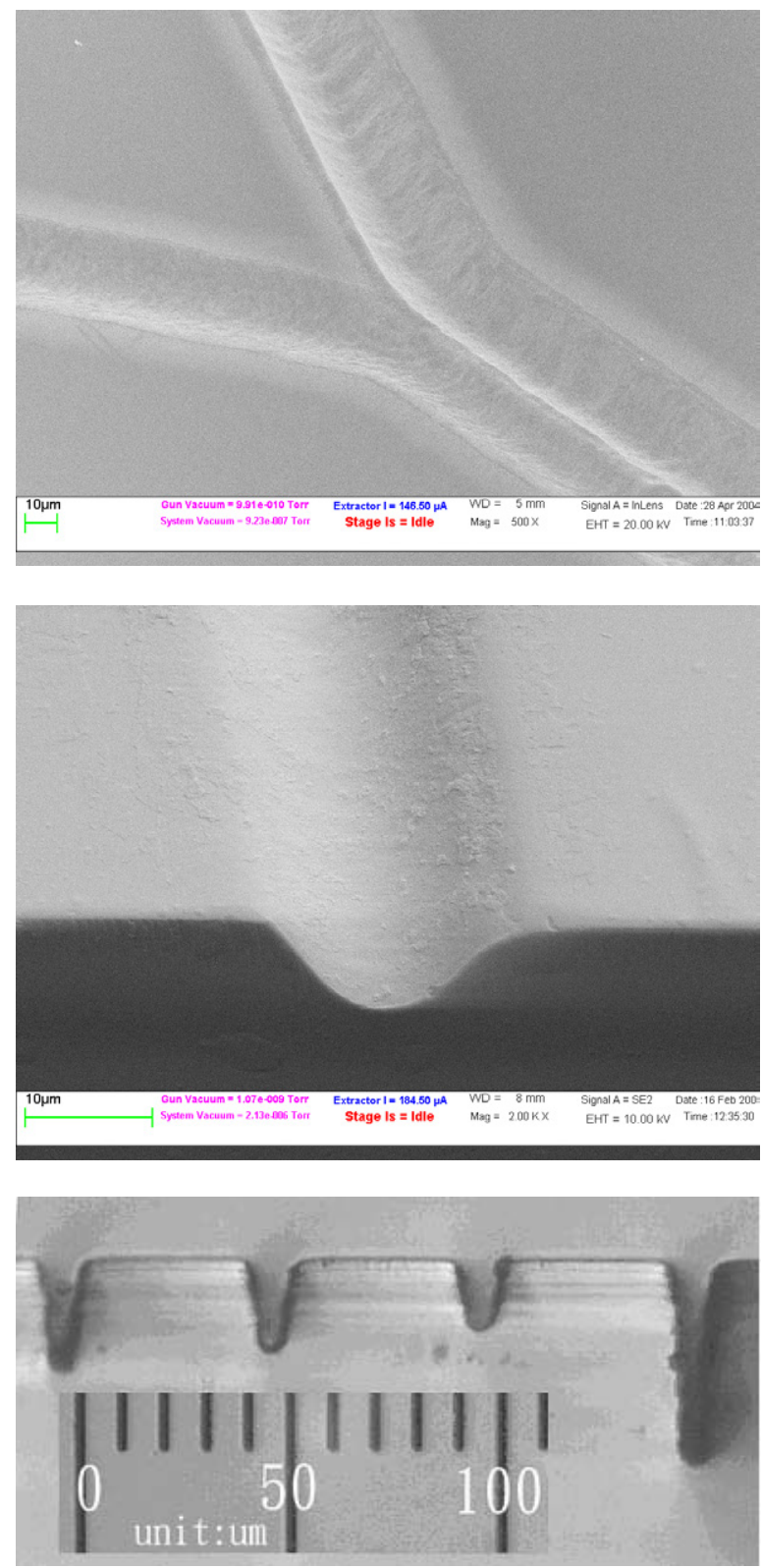

$(e)$ (b)
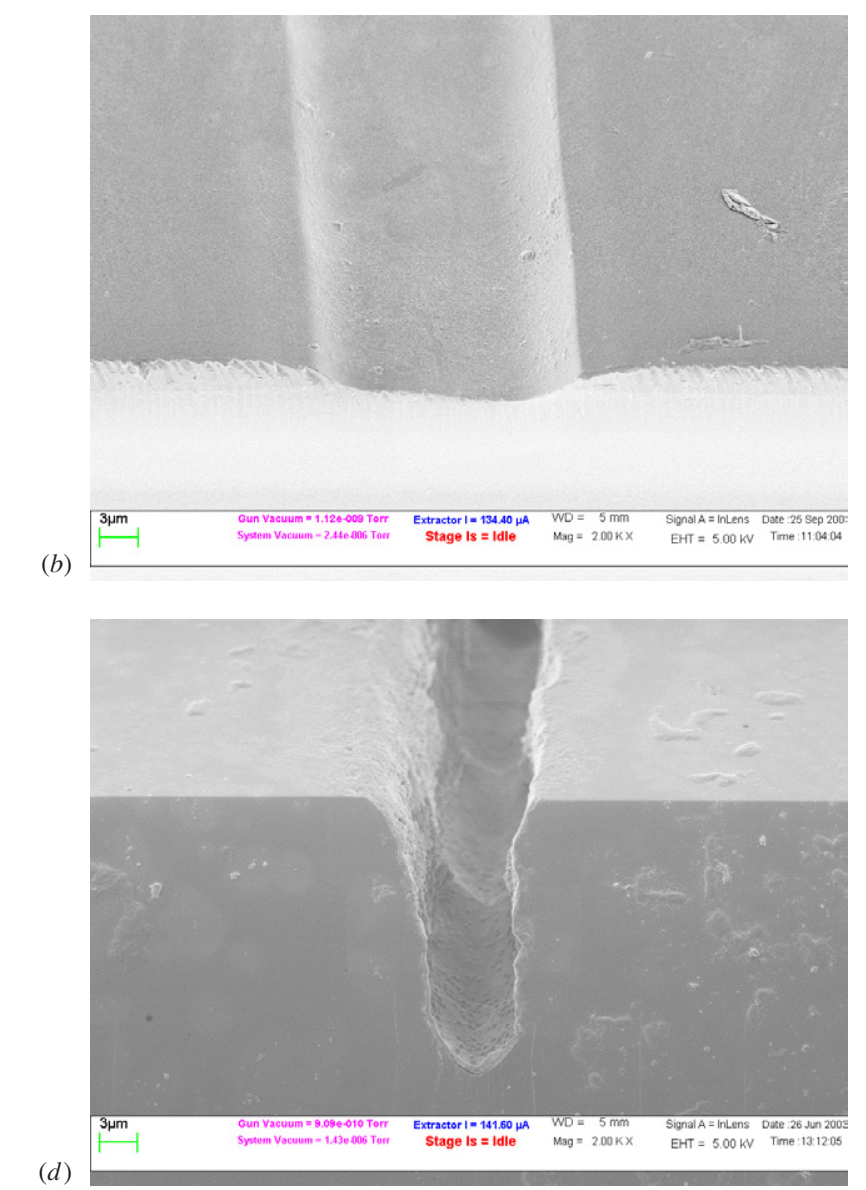

$(d)$

Figure 3. (a) SEM image of two adjacent trenches that merge into an asymmetric trench. The ripple at the bottom of the trench is caused by the speed fluctuation of the CNC stage. The length of the scale bar is $10 \mu \mathrm{m}$. (b) Smooth etching is obtained by optimizing the CNC motion parameter. The trenches in these images are etched with a laser pulse of energy $78 \mu \mathrm{J}$. The length of the scale bar is $3 \mu \mathrm{m}$. $(c)$ SEM image of a $6 \mu \mathrm{m}$ wide trench. The aspect ratio is 0.5 . The image is obtained at a viewing angle of $40^{\circ}$ above the chip surface. The length of the scale bar is $10 \mu \mathrm{m}$. (d) SEM image of a trench with an aspect ratio of larger than two. The width at half height is $7 \mu \mathrm{m}$. The number of scans is 6 . The length of the scale bar is $3 \mu \mathrm{m}$. (e) An optical microscope image of trenches with various aspect ratios in a single chip. The numbers of scans from left to right are 5, 4, 3 and 8 . The laser repetition rate is $4.5 \mathrm{kHz}$ and the average power $35 \mathrm{~mW}$ for all these trenches. The $Y$-axes in $(b)$ and $(d)$ are enlarged to reveal the slightly distorted rim. The scanning speed of the laser beam is $0.2 \mathrm{~mm} \mathrm{~s}^{-1}$, except in $(b)$ and $(d)$ where it is $0.6 \mathrm{~mm} \mathrm{~s}^{-1}$. The etchant is $0.4 \mathrm{M}$ pyrene in acetone.

\subsection{Possible effect of the LIBWE process on electrokinetic phenomena}

Electrokinetic pumping is frequently performed in glass microfluidic channels, such as when a sample is introduced for capillary electrophoresis. The electrokinetic flow depends on the surface properties of the microchannel. Surface element (x-ray photoelectron spectrum) analysis reveals that the glass substrate does not significantly change when pyrene/acetone is used as photosensitizer. However, when another solvent such as tetrachloroethylene $\left(\mathrm{C}_{2} \mathrm{Cl}_{4}\right)$ is used, the surface element contains $\mathrm{Cl}$ [34]. The LIBWE process may therefore affect the electrokinetic properties. This issue is of interest but is beyond the scope of the current study.

\subsection{An integrated microreactor/microconcentrator}

Figure 4 presents a microfluidic system that integrates a microreactor and a microconcentrator to demonstrate further the flexibility of the laser direct-writing method. Figure 4(a) 
shows the design of the system. Figure $4(b)$ presents a photograph. The entire system is filled with dye to increase the image contrast of the microfluidic channel. Direct-writing can be used to prepare the trench pattern in $10 \mathrm{~min}$. The trenched glass chip is then thermally bonded with a blank borofloat glass to form the sealed channel. The trench fabricated by the LIBWE process does not interfere with good thermal bonding, as can be determined by comparison with the bonding of two pieces of blank borofloat glass. The inset in figure $4(b)$ presents a typical bonded cross-section.

The sample chip presented in figure 4 consists of two sections. The first section is a serpentine channel that serves as a microreactor. Reactants are introduced through the two inlets, A and B. The reactants react when they flow through the serpentine section. Air or flowing liquid to be transported in parallel with the crude product from the microreactor is introduced through inlet $\mathrm{C}$. The second section consists of two adjacent trenches with different aspect ratios, as mentioned previously in relation to the transversely asymmetric trench. This section is employed to concentrate or extract the crude product. The final product is collected at port D or E, depending on the actual operation. In the extraction mode, the product is collected at port $\mathrm{D}$, while in the concentration mode, the product is collected at port E.

Figure $4(c)$ shows the fluorescence image of extraction process inside a microchannel similar to the extraction section in figure 4(a). Rhodamine B in water is pumped into the lower channel while a stream of butanol is pumped into the upper channel. The two immiscible streams merge but do not mix. A clear interface is observed between the two phases, indicating stable laminar flow. Rhodamine B extraction from water phase to butanol phase is observed by the fluorescence intensity shift from water to butanol in the downstream region. Although the channel cross-section shown in this example is laterally asymmetric, the structure is not necessary for an extraction process.

The advantage of laser direct-writing is the flexibility of fabrication and the reduced development time. A new microfluidic pattern on the glass chip can be fabricated in 10 min whenever the above design needs to be modified. The development can be completed in 1 day, including the overnight thermal bonding process that involves heating and cooling.

\subsection{High-repetition-rate laser enables high-speed micromachining of glass}

When combined with a high-repetition-rate laser that is used in this study, the direct-writing process is rapid. The repetition rate can be increased to several tens of $\mathrm{kHz}$. For comparison, an excimer laser system typically has a low repetition rate of several tens of Hz. A Q-switched lamp-pumped Nd:YAG usually has a higher repetition rate of approximately $1 \mathrm{kHz}$. The limitation on the increase in the machining speed with the increase in the laser repetition rate is of interest, in particular for the borofloat glass used in this study.

The per-pulse etch rate was measured as the repetition rate increased. Figure 5 presents the results. The laser sweep speed was fixed at $0.2 \mathrm{~mm} \mathrm{~s}^{-1}$. The figure reveals that the etching rate changes only slightly at a repetition rate of below $4 \mathrm{kHz}$.

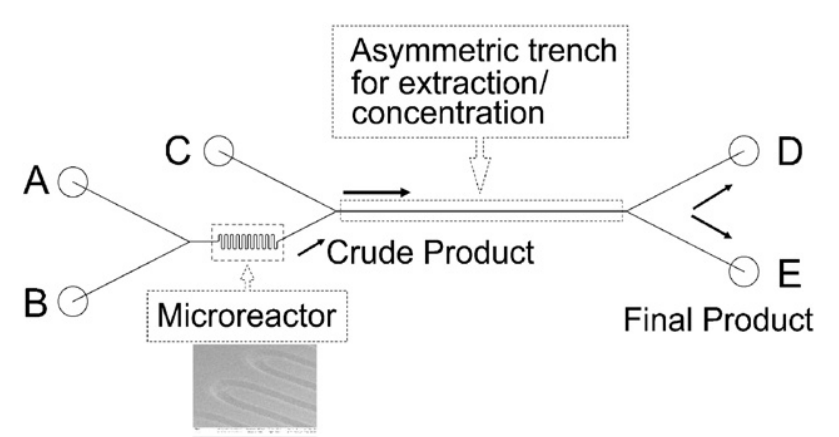

(a)

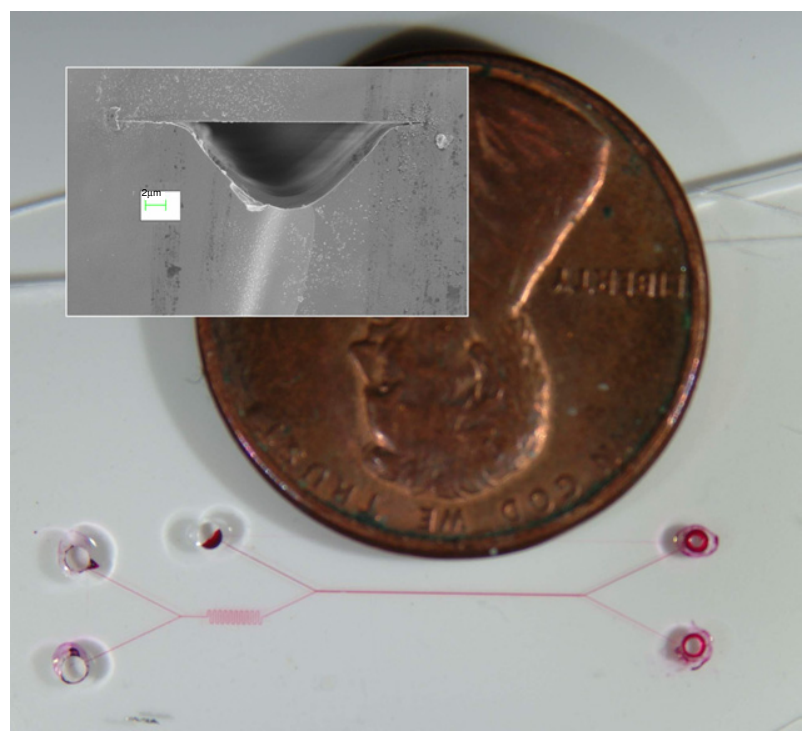

(b)

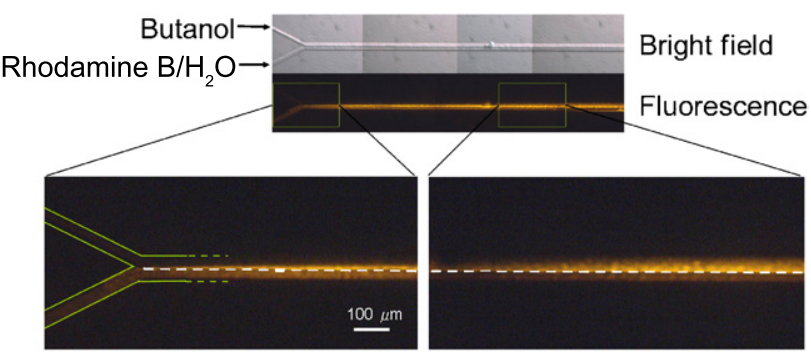

(c)

Figure 4. (a) Designed pattern of a microreactor/concentrator. The inset shows the SEM image of the serpentine region. (b) Photograph of the complete microfluidic system. A one-cent coin is placed next to the device for comparison of sizes. The inset presents a bonded borofloat glass microchannel. The length of the scale bar is $2 \mu \mathrm{m}$. The borofloat chip is etched with laser pulse energy of $78 \mu \mathrm{J}$. The repetition rate is $4.5 \mathrm{kHz}$ and the laser sweep speed is $0.2 \mathrm{~mm} \mathrm{~s}^{-1}$. The etchant is $0.4 \mathrm{M}$ pyrene in acetone. (c) Fluorescence image showing successful extraction from water phase to organic phase. Rhodamine B dissolved in water is pumped into the lower inlet of the extraction channel while clean butanol is pumped into the upper channel. Clear interface indicates stable laminar flow in the emerged section. The extraction is observed by the fluorescence in the butanol phase downstream. The channel width is $46 \mu \mathrm{m}$ in this example. The flow speed is $4 \mathrm{~mm} \mathrm{~s}^{-1}$.

(This figure is in colour only in the electronic version)

The etching rate increases to a maximum of approximately $6 \mathrm{kHz}$ at a repetition rate of above $4 \mathrm{kHz}$. The etching rate declines abruptly as the repetition rate increases above $6 \mathrm{kHz}$. 


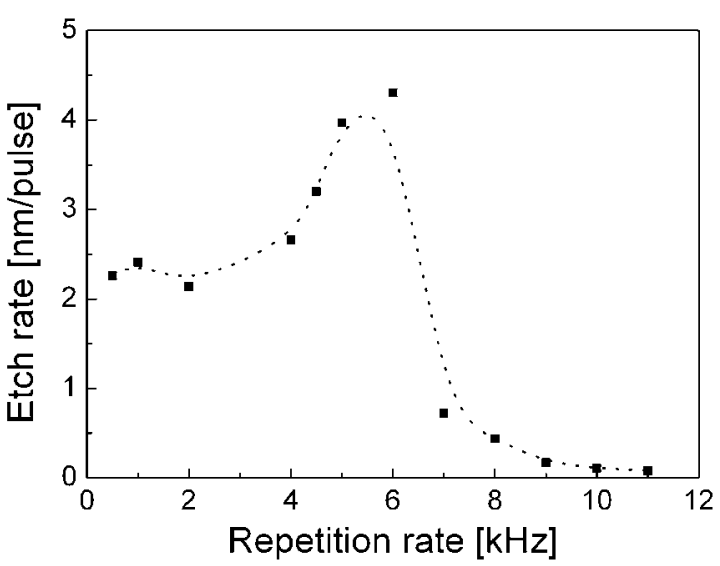

Figure 5. Effect of laser repetition rate on the actual etching rate. The dotted line is a visual guide. The laser pulse energy is $78 \mu \mathrm{J}$. The repetition rate is $4.5 \mathrm{kHz}$ and the laser sweep speed is $0.2 \mathrm{~mm} \mathrm{~s}^{-1}$. The etchant is $0.4 \mathrm{M}$ pyrene in acetone.

At repetition rates of over $10 \mathrm{kHz}$, the laser energy does not result in any observable etching. The findings reveal that the machining speed cannot be increased without limit by increasing the laser repetition rate. An intrinsic factor may impair the etching process at high repetition rate.

Backside glass etching assisted by pyrene solution has been posited to be caused by the propagation of the shockwave from the spot irradiated by a laser [33]. When the pyrene solution absorbs laser energy, a bubble forms at the irradiated spot. According to Ding et al [33], the bubble expands during the first $100 \mu$ s and does not disappear until $140 \mu$ s following the laser pulse. In our study, the etching rate declines sharply as the repetition rate increases above $6 \mathrm{kHz}$, which corresponds to a pulse interval of $\sim 160 \mu \mathrm{s}$. Therefore, when a repetition rate of higher than $6 \mathrm{kHz}$ is used, the intervals between the pulses are too short to allow the bubble to disappear. The bubble can impair contact between the etchant (such as pyrene/acetone) and the substrate, so the etching process is impeded. Indeed, the solvent evaporated but no glass etching was observed when the repetition rate exceeded $10 \mathrm{kHz}$, indicating that the energy of the laser is in fact absorbed by the solution but is not effective in glass etching. The size of the laser beam and the dimensions of the bubbles may affect the bubble collapse time. The optimal repetition rate may vary accordingly. Further work is being conducted to investigate the effect of the beam size.

\subsection{Machining speed of laser direct-writing on borofloat glass}

The reported laser machining technique is very suitable for prototype microtrenching. The overall machining speed can be examined by the following example. Assume that the beam size is $100 \mu \mathrm{m}$ and the expected depth of the trench is $10 \mu \mathrm{m}$. Consider a $4 \mathrm{~nm} /$ pulse etching rate and a $6 \mathrm{kHz}$ repetition rate: the beam sweeping speed should be $0.6 \mathrm{~mm} \mathrm{~s}^{-1}$, as calculated by equation (2). Accordingly, fabricating a $100 \mathrm{~mm}$ long trench with a depth of $10 \mu \mathrm{m}$ takes approximately $720 \mathrm{~s}$. For comparison, the $\mathrm{Ar}^{+}$dry etching rate on borosilicate glass is $0.01 \mu \mathrm{m} \mathrm{min}{ }^{-1}$ [44]. In this example, while omitting the time for photomask generation and photolithographic pattern transfer, the etching speed of backside etching is about two orders greater than that of $\mathrm{Ar}^{+}$ ion etching. Indeed, photolithography is a method that is more appropriate for parallel processing. However, the machining speed on glass is low. Alternatively, a high-end industrial oxide etcher such as Applied Centura eMAX from Applied Materials provides an oxide etching rate of up to $6000 \AA \mathrm{min}^{-1}$. However, the accessibility of such a system is limited for most researchers.

Currently, the trench profile generated by the LIBWE process is Gaussian shape, which is governed by the laser beam profile. In principle, steep wall could be produced by employing flat-top laser beams for machining. This could produce square trench profile that is easier for theoretical flow analysis.

\subsection{Effect of photothermal sensitizer on the etching threshold}

The choice of photothermal sensitizer is not trivial. A compound that has a high extinction coefficient and a high concentration in solution may yield a poor etching rate. For instance, Ding et al used a $1.0 \mathrm{M}$ pyrazine salt solution $\left(\varepsilon_{248} \sim 10000\right)$ and obtained a low etch rate of $0.1 \mathrm{~nm} /$ pulse [33], which reveals that the etching mechanism is not understood completely and that more experimental data are required to improve our understanding of the LIBWE process.

Glass is highly resistant to chemical corrosion. The organic sensitizer used in this study comprised only $\mathrm{C}, \mathrm{H}$ and $\mathrm{O}$, so the product of photolysis or thermolysis does not contain any acidic substance that is as strongly acidic as HF, which is effective in etching glasses.

In the 'liquid hammer' model proposed by Ding et al [33], the photothermal process converts photo energy into thermal energy. The media for this conversion process are the solute and/or solvent molecules, whichever absorbs the photo energy and converts it into thermal energy. Zimmer et al [35] compared the etching of fused silica by $248 \mathrm{~nm}$ and $351 \mathrm{~nm}$ excimer lasers. Three-time extinction at $248 \mathrm{~nm}$ results in a $1 / 3$ etching threshold when pyrene/acetone solution is used. Wang et al [29] also found that increasing the pyrene concentration reduces the etching threshold. These results confirm the role of the solvent and solute in photothermal conversion. Therefore, the etching thresholds of various aromatic compounds were compared to understand better the role of the etchant. Many novel compounds that are effective for the LIBWE process were found. Table 1 lists the results. The first group of compounds are liquids under ambient conditions. The compounds in the second group are all solid powders under ambient conditions and are dissolved in acetone for etching. None of the listed compounds, except for toluene and pyrene, have been reported to be used before in backside glass etching. The lowest etching threshold of these compounds was $\sim 140 \mathrm{~mJ} \mathrm{~cm}^{-2}$ when $1.3 \mathrm{M}$ 9-methylanthracene was used. The corresponding pulse energy was only $31 \mu \mathrm{J}$ for a $30 \mu \mathrm{m}$ wide trench, as presented in figures $2(d)$ and $3(a)$.

The compounds in the first group have thresholds of around $200 \mathrm{~mJ} \mathrm{~cm}{ }^{-2}$, which is close to that of $0.4 \mathrm{M}$ pyrene in acetone. The etching threshold of toluene is comparable to that in the literature [36]. The two compounds, $t$-butylbenzene and 1,2,4-trimethylbenzene, have a lower threshold than does 


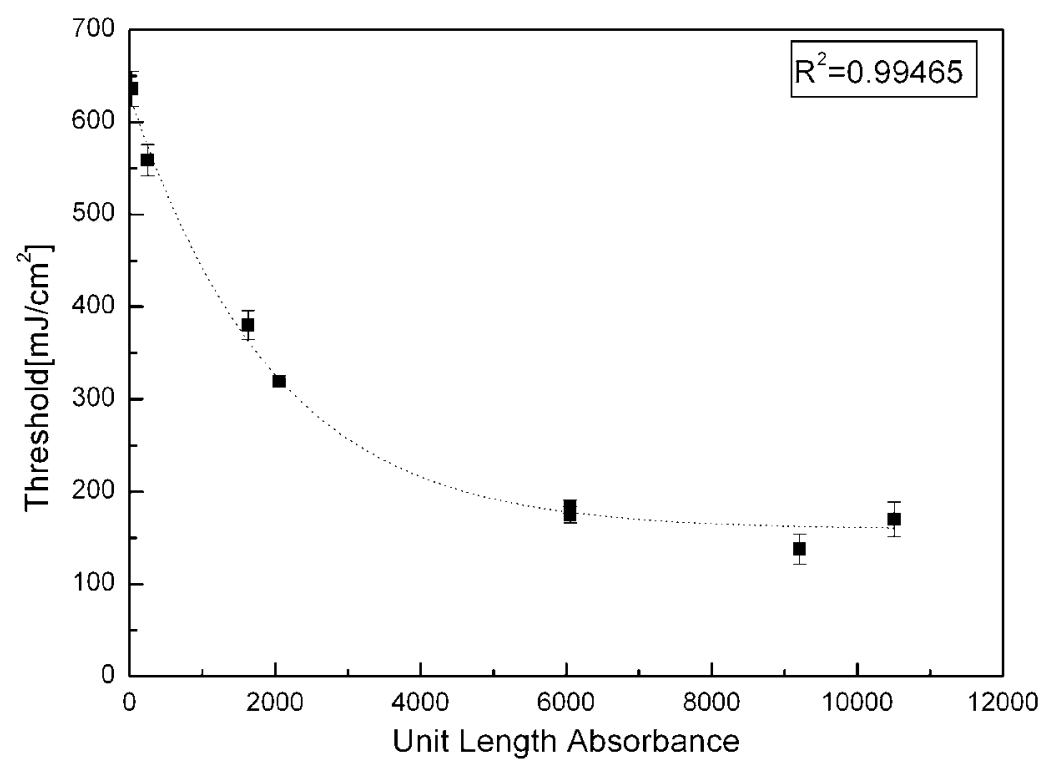

Figure 6. Effect of unit length absorbance on the etching threshold in the acetone solution. The dotted line represents a tentatively fitted exponential decay.

Table 1. Etching characteristics of various aromatic compounds. The first group of compounds above the bold line are organic solvents. They are directly used for etching. The second group, listed below the bold line, are solid compounds. They are dissolved in acetone at the listed concentrations.

\begin{tabular}{llllll}
\hline Compounds & Structure & $\begin{array}{l}\text { Threshold } \\
\left(\mathrm{mJ} \mathrm{cm}^{-2}\right)\end{array}$ & $\begin{array}{l}\text { Concentration } \\
(\mathrm{C})\end{array}$ & $\begin{array}{l}\text { Extinction } \\
\text { coefficient }(\varepsilon)\end{array}$ & $\begin{array}{l}\text { Fluorecence } \\
\text { quantum yield }\end{array}$ \\
\hline Toluene & 239 & 9.4 & Not used & $0.17^{\mathrm{c}}$ \\
t-Butylbenzene & 190 & 6.4 & Not used & $0.14^{\mathrm{c}}$ \\
1,2,4-Trimethylbenzene & 199 & 7.3 & Not used & $0.41^{\mathrm{c}}$ \\
Naphthalene & 319 & 0.4 & $5129^{\mathrm{a}}$ & $0.23^{\mathrm{c}}$ \\
Phenanthrene & 175 & 0.4 & $15136^{\mathrm{a}}$ & $0.13^{\mathrm{c}}$ \\
9nthracene & 636 & 0.04 & $794^{\mathrm{a}}$ & $0.36^{\mathrm{c}}$ \\
9-Methyl anthracene & 138 & 1.3 & $7080^{\mathrm{a}}$ & $0.35^{\mathrm{c}}$ \\
Pyrene & 559 & 0.02 & $12590^{\mathrm{a}}$ & $0.35^{\mathrm{d}}$ \\
Fluoranthene & 184 & 0.4 & $15136^{\mathrm{a}}$ & $0.32^{\mathrm{c}}$ \\
9-Phenyl anthracene & 170 & 1.1 & $9550^{\mathrm{a}}$ & $0.3^{\mathrm{c}}$ \\
\hline
\end{tabular}

$$
\begin{aligned}
& \text { a [45]. } \\
& \text { b [46]. } \\
& \text { c [47]. } \\
& \text { d [48]. }
\end{aligned}
$$

toluene. These compounds differ from toluene in having two more methyl groups. The alkyl side chain is well known to be 'softer' than the benzene ring, so that the vibronic interaction, including the motion of the side chain, is strong for those vibrational modes. Restated, the electronic energy is more prone to dissipate through the vibration of the side chains. For example, the internal conversion factor $\left(k_{\mathrm{IC}}\right)$ of $t$ butylbenzene is ten times greater than that of toluene because the tert-butyl group acts as a 'loose bolt' [49] that accelerates internal conversion. Accordingly, $t$-butylbenzene and 1,2,4trimethylbenzene convert the photon energy more efficiently than toluene, resulting in a lower etching threshold. The threshold-structure relationship of the large analogs of these aromatic solvents in the second group is not as straightforward as that of the aromatic solvents.

Theoretically, the overall efficiency of the photothermal process should be related to the unit length absorbance, $\varepsilon \times[C]$. A larger value corresponds to a higher conversion 
efficiency. Figure 6 depicts the relationship between the etching threshold and the unit length absorbance of the acetone solution. The plot can be nicely fitted with an exponential decay curve. Although the fit is tentative $\left(R^{2}=0.99465\right)$, the plot reveals that the etching threshold reaches a minimum of $\sim 150 \mathrm{~mJ} \mathrm{~cm}^{-2}$ at a unit length absorbance of around 8000 and does not decrease further as the unit length absorbance increases. Zimmer et al made the unusual observation that in pyrene/toluene solution, contrary to that in pyrene/acetone solution, the extinction at $248 \mathrm{~nm}$ that is three times larger than that at $351 \mathrm{~nm}$ results in almost the same etching threshold [35]. Other effect such as fluorescence quantum yield $\left(\Phi_{\mathrm{F}}\right)$ is therefore examined.

The effect of fluorescence quantum yield $\left(\Phi_{\mathrm{F}}\right)$ on the etching threshold is examined as follows. A higher $\Phi_{\mathrm{F}}$ results in weaker non-radiative transition and hence poorer photothermal conversion. However, as presented in table 1, $\Phi_{\mathrm{F}}$ does not correlate well with the etching threshold. Anthracene and naphalene have similar or lower $\Phi_{\mathrm{F}}$ than pyrene but yield higher etching threshold. At high concentration, the fluorescence is quenched by neighboring fluorophore and the radiative energy dissipation is mostly replaced by nonradiative transition. Under such conditions, the absorption and solution concentration dominate the overall photothermal energy conversion efficiency. Hence, the fluorescence quantum yield does not correlate well to the etching threshold. The plot in figure 6 also suggests this correlation. Further work is being conducted to clarify the details of the dependence of the etching rate on the solute concentration for each compound.

\section{Conclusion}

This work describes a rapid prototyping platform for fabricating a crack-free and debris-free glass microfluidic chip. The process does not require a clean room facility or the use of the highly corrosive chemical, HF, for glass etching. A $266 \mathrm{~nm}$ DPSS laser with a high repetition rate is employed and laser-induced backside etching is performed. The microfluidic pattern is drawn using common computer software and the pattern is automatically translated into CNC motion. Without the use of a photomask, a glass microfluidic system can be etched in $10 \mathrm{~min}$. The overall development time is shortened to less than 1 day. A very low laser power (several tens of $\mathrm{mW}$ ) is used and a high laser repetition rate enables swift direct-writing. The use of a low-power laser makes this platform very affordable. The optimal repetition rate was approximately $6 \mathrm{kHz}$ in this work. Smooth etching is observed by a surface profiler and SEM. Complex structures such as stepped trenches and transversely asymmetric trenches were fabricated and characterized. An example with integrated microreactor/concentrator pattern is presented.

Various aromatic compounds are investigated herein and their effects on the etching threshold are compared. Lower thresholds were observed for aromatic solvents with stronger vibronic interactions. Acetone solutions with aromatic solutes have a minimal threshold of $150 \mathrm{~mJ} \mathrm{~cm}^{-2}$. The etching threshold does not decrease further when the unit length absorbance exceeds 8000 .

\section{Acknowledgments}

The authors would like to thank the National Science Council (contract no: NSC93-3112-B-001-013-Y) and Academia Sinica, Taiwan, for their financial support.

\section{References}

[1] Edel J B, Fortt R, de Mello J C and de Mello A J 2002 Microfluidic routes to the controlled production of nanoparticles Chem. Commun. 10 1136-7

[2] Mitchell M C, Spikmans V and de Mello A J 2001 Microchip-based synthesis and analysis: control of multicomponent reaction products and intermediates Analyst 126 24-7

[3] Wootton R C R, Fortt R and de Mello A J 2002 On-chip generation and reaction of unstable intermediates-monolithic nanoreactors for diazonium chemistry: azo dyes Lab. Chip 2 5-7

[4] Wootton R C R, Fortt R and de Mello A J 2002 A microfabricated nanoreactor for safe, continuous generation and use of singlet oxygen Org. Process Res. Dev. 6 187-9

[5] Wilding P, Shoffner M A and Kricka L J 1994 PCR in a silicon microstructure Clin. Chem. 40 1815-8

[6] Northrup M A, Benett B, Hadley D, Landre P, Lehew S, Richards J and Stratton P 1998 A miniature analytical instrument for nucleic acids based on micromachined silicon reaction chambers Anal. Chem. 70 918-22

[7] He P, Haswell S J and Fletcher P D I 2004 Microwave heating of heterogeneously catalysed Suzuki reactions in a micro reactor Lab. Chip 4 38-41

[8] Daridon A, Sequeira M, Pennarun-Thomas G, Dirac H, Krog J P, Gravesen P, Lichtenberg J, Diamond D, Verpoorte E and de Rooij N F 2001 Chemical sensing using an integrated microfluidic system based on the Berthelot reaction Sensors Actuators B 76 235-43

[9] Salimi-Moosavi H, Tang T and Harrison D J 1997 Electroosmotic pumping of organic solvents and reagents in microfabricated reactor chips J. Am. Chem. Soc. 119 8716-7

[10] Daridon A, Fascio V, Lichtenberg J, Wutrich R, Langen H, Verpoorte E and de Rooij N F 2001 Multi-layer microfluidic glass chips for microanalytical applications Fresenius' $J$. Anal. Chem. 371 261-9

[11] Shoffner M A, Cheng J, Hvichia G E, Kricka L J and Wilding P 1996 Chip PCR: I. Surface passivation of microfabricated silicon-glass chips for PCR Nucleic Acids Res. 24 375-9

[12] Cheng J-Y, Wei C-W, Hsu K-H and Young T-H 2004 Direct-write laser micromachining and universal surface modification of PMMA for device development Sensors Actuators B 99 186-96

[13] Lim D, Kamotani Y, Cho B, Mazumder J and Takayama S 2003 Fabrication of microfluidic mixers and artificial vasculatures using a high-brightness diode-pumped Nd:YAG laser direct write method Lab. Chip 3 318-23

[14] Klank H, Kutter J P and Geschke O $2002 \mathrm{CO}_{2}$-laser micromachining and back-end processing for rapid production of PMMA-based microfluidic systems Lab. Chip 2 242-6

[15] Liu Y, Rauch C B, Stevens R L, Lenigk R, Yang J, Rhine D B and Grodzinski P 2002 DNA amplification and hybridization assays in integrated plastic monolithic devices Anal. Chem. 74 3063-70

[16] Giridhar M S, Seong K, lzgen A S L, Khulbe P, Peyghambarian N and Mansuripur M 2004 Femtosecond pulsed laser micromachining of glass substrates with application to microfluidic devices Appl. Opt. 43 4584-9

[17] Buerhop C, Blumenthal B, Weissmann R, Lutz N and Biermann S 1990 Glass surface treatment with excimer and $\mathrm{CO}_{2}$ lasers Appl. Surf. Sci. 46 430-4 
[18] Ihlemann J, Wolff B and Simon P 1992 Nanosecond and femtosecond excimer laser ablation of fused silica Appl. Phys. A 54 363-8

[19] Ihlemann J 1992 Excimer laser ablation of fused silica Appl. Surf. Sci. 54 193-200

[20] Dyer P E, Farley R J, Giedl R and Karnakis D M 1996 Excimer laser ablation of polymers and glasses for grating fabrication Appl. Surf. Sci. 96-8 537-49

[21] Ihlemann J and Wolff-Rottke B 1996 Excimer laser micro machining of inorganic dielectric Appl. Surf. Sci. $106282-6$

[22] Shuttleworth S 1996 Optimisation of laser wavelength in the ablation sampling of glass materials Appl. Surf. Sci. 96-98 513-7

[23] Varel H, Ashkenasi D, Rosenfeld A, Wahmer M and Campbell E E B 1997 Micromachining of quartz with ultrashort laser pulses Appl. Phys. A 65 367-73

[24] Kruger J, Kautek W, Lenzner M, Sartania S, Spielmann C and Krausz F 1998 Laser micromachining of barium aluminium borosilicate glass with pulse durations between $20 \mathrm{fs}$ and 3 ps Appl. Opt. 127-129 892-8

[25] Rudolph P, Bonse J, Kruger J and Kautek W 1999 Femtosecond- and nanosecond-pulse laser ablation of bariumalumoborosilicate glass Appl. Phys. A 69 S763-6

[26] Li Y, Itoh K, Watanabe W, Yamada K, Kuroda D, Nishii J and Jiang Y 2001 Three-dimensional hole drilling of silica glass from the rear surface with femtosecond laser pulses Opt. Lett. 26 1912-4

[27] Schaffer C B, Brodeur A, Garcia J F and Mazur E 2001 Micromachining bulk glass by use of femtosecond laser pulses with nanojoule energy Opt. Lett. 26 93-5

[28] Zhang J, Sugioka K and Midorikawa K 1998 Laser-induced plasma-assisted ablation of fused quartz using the fourth harmonic of a Nd:YAG laser Appl. Phys. A 67 545-9

[29] Wang J, Niino H and Yabe A 1999 One-step microfabrication of fused silica by laser ablation of an organic solution Appl. Phys. A 68 111-3

[30] Wang J, Niino H and Yabe A 1999 Micromachining of quartz crystal with excimer lasers by laser-induced backside wet etching Appl. Phys. A 69 S271-3

[31] Zhang J, Sugioka K and Midorikawa K 1999 High-quality and high-efficiency machining of glass materials by laser-induced plasma-assisted ablation using conventional nanosecond UV, visible, and infrared lasers Appl. Phys. A 69 S879-82

[32] Schafer D, Ihlemann J, Marowsky G and Herman P R 2001 F2-laser ablation patterning of dielectric layers Appl. Phys. A 72 377-9

[33] Ding X, Kawaguchi Y, Niino H and Yabe A 2002 Laser-induced high-quality etching of fused silica using a novel aqueous medium Appl. Phys. A 75 641-5
[34] Bohme R, Braun A and Zimmer K 2002 Backside etching of UV-transparent materials at the interface to liquids Appl. Surf. Sci. 186 276-81

[35] Zimmer K, Braun A and Bohme R 2003 Etching of fused silica and glass with excimer laser at $351 \mathrm{~nm} \mathrm{Appl.} \mathrm{Surf.}$ Sci. 208-209 199-204

[36] Niino H, Yasui Y, Ding X, Narazaki A, Sato T, Kawaguchi Y and Yabe A 2003 Surface micro-fabrication of silica glass by excimer laser irradiation of organic solvent J. Photochem. Potobiol. A 158 179-82

[37] Vass C, Hopp B, Smausz T and Ignacz F 2004 Experiments and numerical calculations for the interpretation of the backside wet etching of fused silica Thin Solid Films 453-454 121-6

[38] Ding X, Yasui Y, Kawaguchi Y, Niino H and Yabe A 2002 Laser-induced back-side wet etching of fused silica with an aqueous solution containing organic molecules Appl. Phys. A 75 437-40

[39] Siegman A E 1986 Lasers (Sausalito, CA: University Science Books)

[40] Oleschuk R D, Shultz-Lockyear L L, Ning Y and Harrison D J 2000 Trapping of bead-based reagents within microfluidic systems: on-chip solid-phase extraction and electrochromatography Anal. Chem. 72 585-90

[41] Ekstrom S, Malmstrom J, Wallman L, Lofgren M, Nilsson J, Laurell T and Marko-Varga G 2002 On-chip microextraction for proteomic sample preparation of in-gel digests Proteomics 2 413-21

[42] Paegel B M, Emrich C A, Wedemayer G J, Scherer J R and Mathies R A 2002 High throughput DNA sequencing with a microfabricated 96-lane capillary array electrophoresis bioprocessor Proc. Natl Acad. Sci. 99 574-9

[43] Goto M, Sato K, Tokeshi M and Kitamori T 2003 Development of microchip-based bioassay system using cultured cells Micro Total Analysis ed M A Northrup, K F Jensen and D J Harrison (CA, USA: Squaw Valley)

[44] Mello A P, Bari M A and Prendergast P J 2002 A comparison of excimer laser etching and dry etching process for surface fabrication of biomaterials J. Mater. Process. Technol. 124 284-92

[45] Friedel R A and Orchin M 1951 Ultraviolet Spectra of Aromatic Compounds (New York: Wiley).

[46] Hirshberg Y 1948 Trans. Faraday Soc. 4 285-9.

[47] Berlamn B 1971 Handbook of Fluorescence Spectra of Aromatic Molecules (New York: Academic).

[48] Schmidt R, Schaffner K, Trost W and Brauer H-D 1984 J. Phys. Chem. 88 956-8.

[49] Turro N J 1979 Modern Molecular Photochemistry (Merlo Park, CA: Benjamin/Cummings) p 172 